\title{
A força-tarefa da psiquiatria do desenvolvimento
}

\section{I ${ }^{1}$ Ana Cristina Costa Lima, ${ }^{2}$ Sandra Noemi Cucurullo de Caponi I}

Resumo: O presente trabalho é uma análise do vídeo institucional do projeto do Instituto Nacional de Ciência e Tecnologia de Psiquiatria do Desenvolvimento para a Infância e Adolescência (INPD - Brasil). O objetivo do artigo é ampliar o debate sobre a verdade científica e os métodos de prevenção de doença mental na infância e adolescência, por meio da análise do material midiático de divulgação do projeto. $\mathrm{O}$ trabalho para prevenir as doenças mentais e diagnosticar precocemente as psicopatologias já foi iniciado (2010) nas escolas particulares e pretende comprovar sua validade para a construção de políticas públicas. A partir de dados de pesquisas epidemiológicas internacionais, está sendo articulada a criação de uma subespecialidade psiquiátrica. Estamos diante de um superdimensionamento do poder de solução dos fatos da vida, por uma medicina psiquiátrica e sua verdade científica, que lança mão de diagnósticos de doenças não manifestadas, baseados em comportamentos, a serem contidos por psicofármacos e orientação de condutas. É um projeto em fase de implantação, que conta com amplo financiamento, público e privado, e credibilidade institucional para sua implantação. É uma medicalização do social e do psíquico, que serve mais à indústria da doença do que à saúde da população.

> Palavras-chave: medicalização, psiquiatria, infância, adolescência, prevenção.

\author{
1 Doutoranda do Programa de \\ Pós-Graduação Interdisciplinar \\ em Ciências Humanas, \\ Universidade Federal de Santa \\ Catarina (UFSC), estágio de \\ Doutorado (bolsista CAPES \\ n³843106) na Université \\ Michel de Montaigne Bordeaux \\ III (França). Endereço eletrônico: \\ lima.anac@gmail.com \\ 2 Professora associada da UFSC \\ pós-doutorado Sênior na EHESS \\ (Paris-França); pós-doutorado \\ na Universidade de Picardie \\ (França); doutorado e mestrado \\ em Lógica e Filosofia da Ciência \\ pela UNICAMP. Pesquisadora \\ PQ-CNPq. Endereço eletrônico: \\ sandracaponi@gmail.com
}


"Força-tarefa" é um termo militar ${ }^{1}$ que a psiquiatria do desenvolvimento está utilizando para nomear a missão da International Association for a Child and Adolescent Psychiatry and Allied Professions (IACAPAP), com o objetivo de disciplinar e/ou controlar as condutas na infância e adolescência, em prol da saúde mental. Não é de hoje que a medicina se apropria de terminologia militar e policial para expressar suas intenções ou nomear suas ações. Podemos lembrar rapidamente de alguns termos tão familiares, como "campanha", que poderia nos remeter à revolta da vacina no Rio de Janeiro, quando a população se rebelou à imposição da vacinação em massa, no início do século XX. Do mesmo modo, o termo "vigilância", inserido no cotidiano de normatizar e fiscalizar, como a vigilância sanitária ou a vigilância em saúde no Brasil, esta que, pode-se dizer, tem suas raízes em uma polícia médica dos séculos XVIII e XIX, em alguns países da Europa, analisadas sob diferentes óticas em Rosen (1980) e em Foucault (1985). Adentremos o teor do projeto, a verdade científica que esses psiquiatras defendem para a realização de uma força-tarefa preventiva na infância, pela saúde mental da sociedade brasileira.

O alvo principal do projeto são as crianças em idade escolar. A tática é infiltrar-se nas escolas e outros aparelhos de institucionalização (pode-se ler disciplinarização) de crianças, incluindo, claro, os serviços públicos de saúde, em uma tentativa de diagnosticar precocemente a população em risco de ser acometida por uma doença mental. O trabalho para prevenir as doenças mentais e diagnosticar precocemente as psicopatologias foi iniciado em 2010 nas escolas particulares, enquanto nas instituições públicas ele é um pouco mais moroso, pois depende de construção de políticas públicas.

A máquina está sendo financiada pela indústria. Os grandes laboratórios financiam as pesquisas e desenvolvem os medicamentos, definem a educação continuada dos médicos em congressos, bem como aparecem no patrocínio ou apoio de cartilhas e outros instrumentos educacionais (ANGELL, 2007). A psiquiatria oferece como recurso terapêutico central a prescrição de medicamentos, especificamente os psicofármacos. Como sabemos, a indústria financia e visa ao lucro, como é apresentado na declaração de conflitos de interesse que aparece no pequeno texto Força-tarefa brasileira de psiquiatras da infância e adolescência (MORAES et al., 2008). Os critérios utilizados pelo complexo médico- 
industrial, incluídas as instituições de pesquisa, classificam a relação do autor da pesquisa com duas indústrias de medicamentos - que pagam o palestrante, pesquisas e educação continuada do médico - como um conflito de interesses modesto. Há avaliação externa ou a avaliação de conflito de interesse é somente uma formalidade? Voltemos ao nosso foco principal.

Segundo Rohde (2000), o projeto da psiquiatria do desenvolvimento vem sendo construído no Brasil há pelo menos uma década. $\mathrm{O}$ texto citado mais acima é uma das primeiras comunicações desse grande projeto de psiquiatrizar a infância no país. A partir de dados de pesquisas epidemiológicas internacionais, aceitos pela Organização Mundial de Saúde (OMS), é justificada a necessidade da subespecialidade em psiquiatria para a formação do psiquiatra para a infância e adolescência.

Os autores defendem a criação de uma área de concentração para atender a essa faixa etária, especializada no desenvolvimento normal e na avaliação diagnóstica, como a dinâmica e a psicopatologia familiar e a forma de comunicação de crianças. Ainda, afirmam que a comorbidade nestas é significativamente maior do que nos adultos, e que há diferenças importantes na terapêutica psicofarmacológica. É possível destacar duas preocupaçóes ao se criar uma subespecialidade chamada psiquiatria do desenvolvimento ou da infância e da adolescência: a defesa institucional (de reserva de saber e de mercado) e a conceituação de uma teoria das condutas e da prevenção de doenças mentais.

O projeto encontra seu fundamento na hipótese não comprovada de que existiria uma lesão cerebral como causa biológica da(s) doença(s) mental(is) ou em uma linguagem mais atual, um desequilíbrio em neurotransmissores e genes. Porém, de fato, são os dados epidemiológicos que definem a verdade sobre a patologia, sem uma nosologia e/ou uma etiologia clara da doença. A demarcação patológica é definida por alguns critérios abrangentes, especificados no "DSMIV-TR, Manual Diagnóstico e Estatístico de Transtornos Mentais” (2002), e utilizados para pesquisas epidemiológicas e diagnósticos preventivos.

Ainda, é interessante que, ao mesmo tempo em que defende os dados epidemiológicos, o texto coloca em dúvida sua fidedignidade. Ao mesmo tempo em que se utiliza do resultado de uma alta prevalência de doenças mentais, cita alguns diagnósticos absurdos, ambos advindos de pesquisa epidemiológica, como segue: 
Por exemplo, Assumpção e Carvalho (Assumpção FB, Carvalho LN. Realidade do diagnóstico em psiquiatria infantil no Brasil. J. Bras. Psiquiat., no prelo), ao revisarem os dados referentes à morbidade psiquiátrica hospitalar do SUS, segundo os critérios diagnósticos do CID-9 em jovens brasileiros entre 0 e 19 anos de janeiro de 1992 até dezembro de 1997, encontraram 73 bebês com menos de 1 ano diagnosticados como portadores de quadros psicóticos senis e pré-senis, e 2.120 bebês na mesma faixa etária com diagnóstico de psicose esquizofrênica. Os dados não necessitam de discussão - falam por si sós (ROHDE, 2000).

E, assim, o artigo, na intenção de defender a necessidade de formação específica em psiquiatria da infância e da adolescência, é finalizado com um exemplo que mostra que os dados de pesquisa não são, necessariamente, representativos dos agravos, doenças, transtornos psíquicos das pessoas, mas tão somente o resultado de dados de prontuários baseados em manuais de doenças mentais, preenchidos de forma no mínimo displicente.

Desse movimento inicial, como podemos ver na nota abaixo, foi criado o Instituto Nacional de Ciência e Tecnologia de Psiquiatria do Desenvolvimento para a Infância e Adolescência (INPD):

O Instituto Nacional de Ciência e Tecnologia de Psiquiatria do Desenvolvimento para a Infância e Adolescência (INPD) realizou nos dias 19 e 20 de março o seu evento inaugural...

O INPD integra um projeto do Conselho Nacional de Desenvolvimento Científico e Tecnológico (CNPq) que disponibilizou o maior aporte de recursos já destinado para a área de pesquisa no Brasil ( $\mathrm{R} \$ 520$ milhōes). As verbas foram divididas entre os 101 Institutos Nacionais de Ciência e Tecnologia (INCT) aprovados para participar do programa. Na edição de janeiro, o jornal "Psiquiatria Hoje" realizou cobertura especial sobre os INCT...

O projeto terá duração de cinco anos. Inicialmente, está assegurado o financiamento para os três primeiros; após avaliação, será decidida a continuidade do apoio por dois anos adicionais.

Além do IPq [Instituto de Psiquiatria do Hospital das Clínicas da Faculdade de Medicina da Universidade de São Paulo], o INPD tem profissionais nas Universidades Federais de São Paulo (Unifesp), do Rio Grande do Sul (UFRGS), da Bahia (UFBA), de Pernambuco (UFPE) e do Rio de Janeiro (UFRJ), além da Universidade Presbiteriana Mackenzie, da Federal de Santa Maria, da Universidade Metodista (RS), e da Universidade Estadual Paulista Júlio de Mesquita Filho (Unesp). Centros de pesquisa internacionais, como as universidades de Yale, do Texas, Harvard, Duke (Nova Iorque), John Hopkins, da Califórnia em San Diego (UCSD), da Califórnia em Los Angeles (UCLA) e o Instituto de Psiquiatria de Londres, também colaboram com o INCT (Instituto Nacional de Ciência e Tecnologia. (INPD, 2009) 
O INPD disponibiliza na internet um vídeo institucional de apresentação do projeto de intervenção da psiquiatria do desenvolvimento (INPD, s. d.). ${ }^{2}$ O Núcleo de Estudos em Filosofia e História das Ciências da Saúde - UFSC (CNPq) apresentou o vídeo, seguido de um debate, como atividade final do Seminário "A vida medicada: ética, saúde pública e indústria farmacêutica (CAPONI et al., 2010), em agosto de 2009. Segue uma transcrição do vídeo e alguns comentários.

\section{A mídia persuasiva}

A primeira imagem do vídeo é o globo terrestre, um zoom aproxima uma cidade, para, e volta a abrir no globo, com a América do Sul em primeiro plano. O áudio, com uma voz de homem, afirma que: "O Brasil possui hoje cerca de 90 milhōes de crianças e jovens com menos de 18 anos de idade. Dessa população, nada menos que $10 \%$ apresentam algum tipo de doença mental". Uma pequena pausa e o alento "Calma! O mundo não está ficando louco." Nesse ponto, o globo gira em sentido anti-horário e passa para uma foto de Einstein, com a legenda "espectro autista”. A voz nos diz: "Ao contrário do que se pensa, as doenças mentais não estão associadas à loucura ou a algum estereótipo parecido. Qualquer distúrbio ou transtorno de comportamento, personalidade e humor, por exemplo, pode ser um problema mental, e isso não impede as pessoas de levarem vidas normais e até passarem despercebidas no nosso dia a dia”.

Enquanto isso, as fotos são apresentadas, cada uma com sua legenda diagnóstica: Roberto Carlos, "transtorno obsessivo-compulsivo"; Van Gogh, "transtorno bipolar", Steven Spielberg, "hiperatividade" e James Dean, "transtorno de conduta". A informação audiovisual, mesmo nesse caso em que a qualidade técnica é praticamente a de um vídeo caseiro, é sempre impactante. A utilização de recursos de convencimento, como os da publicidade, com afirmações diagnósticas taxativas dão sim, ao contrário do que fala o locutor, a impressão de estereótipos de doenças mentais, que rotulam pessoas famosas.

A primeira informação em áudio é uma afirmativa grave, a de que $10 \%$ da população com menos de 18 anos tem ou vai ter doença mental. Na sequência, informaçôes desajustadas - que doente mental não é louco e que pessoas famosas em várias áreas foram ou são doentes mentais, bem como que é normal ser doente mental, pois "qualquer transtorno de comportamento, personalidade e 
1320 humor pode ser uma doença mental” -, no mínimo deixam quem assiste ao vídeo confuso, o que pode funcionar como um recurso de intimidação.

Essa técnica de comunicação utilizada é persuasiva, um recurso corrente em publicidade e marketing, onde a meta é convencer o público de que ele tem uma necessidade que não tinha antes (MALDONATO, 2004, p. 171-178). Esse recurso, advindo de uma psicologia bastante questionável por suas intenções de manipulação do pensamento e desejos do indivíduo, chama a atenção ao ser utilizado por uma instituição médica para convencimento da população da necessidade de seus serviços. $\mathrm{O}$ recurso serve para captar a atenção e ao mesmo tempo introduzir uma verdade positiva, a fim de convencer a audiência de que o conjunto é verdadeiro.

Segue uma cena de rua, com pessoas indo e vindo, e, à imagem, é sobreposto um esquema de figuras humanas, seguido de 'pizzas' usadas em demonstrativos estatísticos, com as indagações: "E então nos perguntamos: o que está sendo feito por esses nove milhões de jovens no Brasil? Que futuro eles terão, qual será o custo de tratá-los? Qual será o custo social em não tratá-los? E, afinal, quais são esses problemas?” Feita essa introdução, o vídeo é orientado pelas palavras de médicos psiquiatras responsáveis pelas pesquisas e projeto em implantação, como segue:

Estudos têm mostrado, claramente, que transtornos mentais que são identificados de forma transversal na idade adulta, têm o seu início na infância e adolescência. Se nós conseguirmos detectar esses quadros na infância e adolescência, nós podemos estar fazendo algo preventivo em termos da idade adulta. Mais do que isso, a psiquiatria trabalha hoje também com a noção de crianças em risco para o desenvolvimento de transtornos. Esse Instituto vai poder trabalhar, alavancar a saúde mental de crianças, dentro dessa concepção de psiquiatria do desenvolvimento. E, ao mesmo tempo, vai permitir capacitar profissionais da área de saúde mental e professores para noções de saúde mental na infância e adolescência.

A credibilidade da fonte é fundamental em publicidade e marketing, quanto maior a credibilidade da fonte, maior a força sugestiva do material veiculado (MALDONATO, 2004, p.175-6). O médico é uma das profissões de maior credibilidade na sociedade contemporânea ocidental, e, aqui, ele é o comunicador, aquele que anuncia a realidade e nos coloca em cena: eu, você, qualquer um pode ser uma criança ou um adulto com doença mental - o risco é generalizado. Mais, os médicos se apresentam com capacidade para tratar a população e prevenir as doenças antes delas se manifestarem (torna doença o que ainda não é doença: a chave é o ainda). Cria-se uma expectativa sobre o impacto da doença "transtorno 
de comportamento, personalidade e humor" e ao mesmo tempo um temor de contrair o mal. Por outro lado, tranquiliza, oferecendo uma solução para a saúde mental das crianças. Seria pelo controle de condutas indesejáveis? Ou pela diagnose preventiva de crianças em risco? O que aconteceria a essas crianças? Seriam tratadas pela doença que ainda não desenvolveram? É razoável que se altere o metabolismo com drogas medicamentosas como medida preventiva a um comportamento indesejado? E este é indesejado por quem, para quem? Fique claro que é a cura do que ainda não se manifestou, uma cura preventiva do não manifestado.

É introduzido o protagonista, o médico psiquiatra, especialista em desenvolvimento, em infância e adolescência. Ele repete a informação de que os transtornos mentais começam na infância, esse é o conteúdo a ser inculcado na cabeça das pessoas, da população, por inúmeras repetições. Prevenção de doença e detecção de risco aqui são a mesma coisa. Na lógica de que a criança é o futuro adulto, apresenta a proposta, o produto: um trabalho preventivo. A criança não existe em si, é somente um vir a ser, o futuro adulto. $\mathrm{O}$ risco não é para a criança, mas de vir a ser um adulto doente e perigoso, desviante da conduta adequada.

Esse é o projeto: capacitar professores e profissionais de saúde para identificar as crianças em risco de desenvolver transtornos mentais e aquelas que já apresentam alteraçôes de comportamento e, portanto, já estão doentes mentais.

Mas vamos adiante, na análise dos recursos de comunicação. Seguem imagens, que parecem estrangeiras, de um delivery estadunidense, provavelmente, em que um garoto com casaco e capuz aponta uma arma e realiza um assalto, junto com o áudio:

Isso quer dizer que boa parte dos distúrbios e transtornos de pessoas adultas foram desenvolvidos nos primeiros anos de vida, entre 3 e 12 anos". Acabado o assalto, com a chegada da polícia, entra um cérebro na tela e o locutor nos informa que "sabendo identificar e tratar esses problemas, a probabilidade de cura é muito grande, já que depois da doença manifestada é muito difícil uma recuperação total.

Está feita a associação de ladrão, bandido, assaltante, crime com doença mental. No Brasil, como no mundo, há milhões de pessoas que vivem à margem social e psicologicamente, em ambientes de crime. Agora se apresenta um conhecimento científico capaz de afirmar que tudo isso é doença, e que a solução estaria na medicina.

O que parecia ser um problema social em uma sociedade tão desigual se reduz a uma explicação biológica e cerebral. Trata-se de uma estratégia cara ao discurso 
médico, conhecida como medicalização do social, que já estava presente nos anos 1830, com o primeiro projeto médico de saúde pública, analisado por Roberto Machado. A psiquiatria no Brasil teve início com a fundação do primeiro hospício, em 1852 (ODA; DALGALARRONDO, 2005; MACHADO et al., 1978), e a articulação para a construção do manicômio foi uma das primeiras açôes da Sociedade de Medicina e Cirurgia do Rio de Janeiro (fundada em 1829). A psiquiatria, historicamente no país, atendeu aos loucos, afirmando, pelo menos por um século, que o isolamento social seria parte integrante da terapêutica.

É no seio da medicina social que se constitui a psiquiatria. Do processo de medicalização da sociedade, elaborado e desenvolvido pela medicina que explicitamente se denominou política, surge o projeto - característico da psiquiatria - de patologizar o comportamento do louco, só a partir de então considerado anormal e, portanto, medicalizável. (MACHADO et al.,1978, p.376)

Continuando o vídeo, aparece outro psiquiatra que nos afirma:

Existem alguns estudos já brasileiros mostrando a quantidade. Contam quantas crianças teriam algum tipo de problema da ordem do comportamento ou da ordem da saúde mental, como a gente fala. Esses estudos tiveram uma prevalência, quer dizer, uma taxa, uma frequência de aproximadamente de $10 \%$ a $12 \%$ das crianças brasileiras apresentam algum tipo de problema em termos de saúde mental. Isso significa em torno de 9 milhôes de crianças brasileiras tendo algum tipo de problema que interfira no seu desenvolvimento. Até o momento não existem políticas claras, bem definidas, de como encaminhar essa questão. Você imagina, aí, que considerando essas frequências, aproximadamente 4 milhões de brasileiros preenchem critérios para déficit de atenção... E, óbvio, uma criança que tem uma dificuldade de se manter academicamente em progresso, ela tem a chance maior de sair do sistema escolar.

O projeto reivindica a necessidade de a medicina criar a subespecialidade psiquiatria da infância e adolescência, para que faça a gestão dos riscos e defina e trate precocemente a população, para evitar doenças mentais. Precocemente, tanto na idade dos afetados pacientes como no determinismo de condutas de risco. A pretensão é adentrar pelo ensino fundamental, de modo a alcançar toda a população na faixa etária dos 6 aos 15 anos. Sem dúvida, uma combinação de dispositivos disciplinares, que dominem o indivíduo, e uma tática biopolítica, que defina uma prática generalizada, com base em dados estatísticos da epidemiologia.

É uma estratégia de biopoder e de disciplinarização dos corpos, como podemos encontrar nas pesquisas e reflexões de Foucault $(2007,2008)$, em sua genealogia da instituição do Estado moderno e processo de industrialização, nos séculos XVIII e XIX, na França e em outros países da Europa. Aqui, a estratégia de biopoder se 
manifesta por meio da confirmação da doença pelos dados epidemiológicos, que possibilitam a criação de uma verdade científica sobre uma prevalência de doença mental na sociedade e suas consequências sobre o indivíduo. E a disciplinarização se apresenta na intervenção no sistema educacional.

$\mathrm{Na}$ sequência, aparecem crianças na escola e o áudio nos informa:

Crianças começam a apresentar sintomas de distúrbios e transtornos desde a primeira infância, e identificar esses sintomas é imprescindível para o encaminhamento e tratamento correto. A partir dos 3 anos de vida, sintomas como atraso na fala, isolamento social, movimentos repetitivos com as mãos, os chamados flapping, podem ser um indicativo de transtorno de desenvolvimento e comunicação, um problema que atinge 900 mil jovens no Brasil.

Quanto mais precoce o diagnóstico, menor o risco de adoecer. E identificar esses sintomas, criando confusão entre o áudio e a imagem - a criança que está na tela é ainda um bebê de colo e faz um movimento que quase todo bebê faz -, seria uma solução para esclarecer a necessidade de se tratar o que ainda não se manifestou? Por cima da imagem de crianças, sempre uma 'pizza' de dados estatísticos, indicativa de percentual de acometimento e o nome do transtorno. Novamente a criança é de faixa etária abaixo da citada no áudio. Segue o áudio:

Entre os 5 e 8 anos, sintomas como ter muito medo de ir à escola, ser extremamente preocupado, ter dificuldade para dormir, ter muitas manias, esfregar e lavar as mãos, constantemente, são indicativos de transtornos de ansiedade e transtorno compulsivo-obsessivo, o TOC. A prevalência desse problema no Brasil chega a mais de $7 \mathrm{mi}$ lhões de jovens. Outros sintomas presentes na idade entre 5 e 8 anos são estar sempre no mundo da lua, ser extremamente agitado, agir sem pensar, não conseguir parar quieto, como o constante tamborilar dos dedos enquanto realiza outras ações. Essas ações podem identificar transtornos e déficits de atenção e hiperatividade, o TDAH, problema que atinge quase 5 milhões de jovens no Brasil. Problemas de leitura e escrita são percebidos em crianças entre 8 e 10 anos e são indicadores de transtornos de aprendizagem e dislexia, o mais comum na população brasileira. Acredita-se que 9 milhões de jovens brasileiros apresentam esse problema. Problemas da regulação do humor, como irritabilidade ou tristeza e manias em excesso podem representar uma alteração do humor.

$\mathrm{Na}$ sequência de imagens de crianças e depois adolescentes, estes mais agressivos, aparece um rapaz de capuz, de costas, esperando o metrô (que bem poderia ser aquele do assalto mostrado anteriormente). "Crianças e adolescentes que passam a se isolar, mudam o padrão de sono, para mais ou para menos" - a imagem na tela é de um adolescente abrindo um armarinho lotado de frascos de medicamentos e depois tomando muitos comprimidos. E o áudio continua: 

exaltadas, às vezes muito desanimadas, ou começam a falar em morte, podem estar apresentando um transtorno do humor, seja depressão ou mesmo um quadro bipolar.

E, novamente, aparece o rapaz pegando drogas medicamentosas no armarinho do banheiro.

A sequência de ações é ilustrada com crianças, por vezes de faixa etária diferente daquela à qual se refere o texto: seria um engano, simplesmente, ou um recurso técnico? A técnica de repetição, aliada às informações de imagem e texto desconectadas, pode impedir a reflexão de quem assiste e, dessa forma, funcionar como um recurso psicológico de convencimento. Da informação de que a criança insegura está tão próxima de um comportamento agressivo na adolescência, segue a tela para nos apresentar crianças e adolescentes em sofrimento, de um ponto de vista da doença, e não do desenvolvimento cognitivo ou do sofrimento psicológico, mas de um ponto de vista do risco de delinquir, a partir de sintomas isolados, sem contexto psicológico, social e cultural.

É interessante notar, como destaquei na descrição do vídeo, que por duas vezes um adolescente abre um armarinho de parede, típico de banheiro, cheio de medicamentos. Sobre isso não há qualquer referência no áudio, mas o que podemos pensar a respeito, que mensagem é essa? Se a sugestão é de que é comum uma casa ter excesso de medicamentos, parece que a responsabilidade é da indústria e de uma medicina associada à difusão do medicamento. E qual o motivo de apresentar essa imagem? A imagem do menino enchendo a mão de comprimidos do armário lotado deles aparece como uma clara referência ao problema da automedicação, ao fato de que indústria farmacêutica e o saber médico se nutrem de uma forte e necessária aliança. Porém, a imagem não é comentada, a questão é mostrada sem qualquer alusão a como essas drogas medicamentosas estão ao alcance da população e seu uso abusivo, no caso, pelo adolescente.

O vídeo continua com as palavras de outro psiquiatra envolvido no projeto, que explica:

Em primeiro lugar, é preciso ter a noção de que os sintomas mentais começam na infância; esse é o primeiro ponto. O segundo ponto importante é que os transtornos mentais são transtornos do desenvolvimento cerebral. Depois que a doença começa e ela se cronifica - a maior parte dos transtornos mentais se cronificam - a gente tem pouco $[\ldots]$ da recuperação total. $\mathrm{O}$ que nós conseguimos hoje é uma recuperação parcial desses transtornos que se cronificam. A ideia aqui é que a nossa intervenção passe a ser antes da doença começar. 
A nova e definitiva informação: o problema está no cérebro. Tanto faz onde e como se vive, o problema é no cérebro. Um defeito, uma má formação, uma herança genética? Não importa, o que vale é saber que a psiquiatria pode agora resolver esse problema e a sociedade, cada indivíduo, poderá ser curada de sua doença mental, por meio de psicofármacos auxiliados por uma ortopedia das condutas. Claro, se o tratamento for iniciado antes de a doença se manifestar.

Ao se definir como um problema de ordem cerebral, torna-se inquestionável que o poder está nas mãos dos cientistas, que treinam os médicos especialistas para enxergar no comportamento o problema cerebral. Este, por sua vez, não pode ser detectado por qualquer exame clínico ou com recurso de máquinas que vejam o corpo por dentro ou analisem seus fluidos. É preciso crer nas fontes, por meio da palavra do médico psiquiatra.

Nesse ponto, o vídeo passa a dar informações sobre as instituições que promovem as pesquisas e o projeto de intervenção, acompanhadas das respectivas logomarcas:

O INPD nasce de uma parceria entre o Instituto de Psiquiatria da Faculdade de Medicina da USP, Universidade Federal do Rio Grande do Sul e Universidade Federal de São Paulo, a partir de um projeto financiado pelo CNPq. E os seus principais objetivos são:

1. Desenvolver um método de identificação precoce de problemas mentais em crianças e adolescentes.

Sobre este, o psiquiatra explica que:

No primeiro estudo nós vamos coletar sintomas subclínicos, indivíduos que não desenvolveram a doença ainda, mas tem sintomas subclínicos. Onde nós vamos tentar preparar os pais para que eles possam ter atitudes mais adaptativas em relação aos sintomas de seus filhos. Sempre dentro desse objetivo de que eles não desenvolvam a doença.

A identificação precoce se dá por meio de busca de sintomas subclínicos de algum mal que não seja uma patologia orgânica, apesar da afirmação de que se trata de doença do cérebro. Por que não são feitos exames de imageamento e exames complementares de laboratório para definir uma patologia? É aceitável cientificamente que sejam criados protocolos de diagnóstico e intervenção clínica de candidatos a doentes mentais em um tempo futuro?

Um detalhe que não pode passar em branco: não se coletam sintomas, a coleta é de material de pesquisa epidemiológica e sintomas são expressões da pessoa que o profissional de saúde identifica como alguma patologia, a quem chamam 
de sintomas subclínicos. Desse modo, é possível que, potencialmente, qualquer pessoa possa ser sujeito de pesquisa sem o saber.

Continuando com os objetivos, o segundo prega a "formação de médicos psiquiatras sob essa nova perspectiva."

A educação continuada sugere que o conhecimento segue uma linha evolutiva, de compreensão positiva do saber.

E a palavra do médico:

Aqui o objetivo é formar o novo psiquiatra, o novo médico, com essa visão do desenvolvimento. A ideia de que os transtornos mentais são transtornos do desenvolvimento. E que a partir do conhecimento das diversas trajetórias ao longo do desenvolvimento, nós vamos identificar aquelas trajetórias atípicas e testar intervenções para trazer esse indivíduo de volta para uma trajetória típica.

Seguem os objetivos. Dessa vez, o terceiro deles recomenda a "transmissão desse conhecimento à população através de professores e profissionais do Programa de Saúde da Família (PSF).”

O psiquiatra esclarece:

E aqui a grande ênfase vai ser no professor. A ideia é criar uma tecnologia que permita ao professor detectar precocemente pequenos sintomas prodômicos. Ele, então, vai encaminhar essas crianças para o Programa de Saúde da Família, que também vai estar habilitado, capacitado, a partir de uma tecnologia apropriada para detectar e diagnosticar precocemente, essas crianças e encaminhar para a rede secundária, que vai se tornar, então, uma rede protetora e não uma rede que só trata do indivíduo doente. Uma rede que vai tratar do indivíduo que ainda não adoeceu.

Parece que cabe ao professor, além de disciplinar, denunciar aqueles que aparecem com desvio da normalidade, cuja conduta indica um futuro (provável) adolescente delinquente. Os portadores de tais sintomas subclínicos, detectados por professores e médicos treinados pelos psiquiatras especializados em infância e adolescência, poderão ser medicados, por até toda a vida. Serão tratados pela doença que não têm e que não apresenta qualquer indício orgânico de patologia. A defesa da verdade para a instauração da práxis clínica é de que todos os recursos tecnológicos, que mostram a credibilidade, serão utilizados.

A propósito do quarto objetivo, "[d]ifusão dessas informaçōes através da telemedicina”, o psiquiatra esclarece que:

Nós vamos tentar educar diferentes professores em áreas mais distantes, formar os novos médicos nessa nova perspectiva também, nas áreas mais distantes, nos quatro cantos do país e, por último, nós estamos trabalhando para trazer um prontuário eletrônico, 
que não só identificamos como o melhor prontuário eletrônico que existe na atuali-

dade. E daí, uma vez traduzido, implementado e adaptado à nossa realidade, vamos levá-lo para a Universidade Federal do Rio Grande do Sul, de São Paulo, da Bahia, de Pernambuco e vamos fazer um projeto piloto e ver se nós conseguimos coletar dados a partir desse prontuário eletrônico, em conjunto com todas essas universidades.

O esforço, a verdadeira força-tarefa para solução dos problemas de uma sociedade, defendidos como doenças mentais e enfrentados por meio da medicalização da infância e adolescência, seria uma normalização de condutas e utilização de drogas que alteram o comportamento, com recurso de alta tecnologia para a disciplinarização de médicos, professores, crianças e pais.

Por fim, o vídeo fecha com a seguinte afirmação:

Na verdade, o que nós esperamos do projeto é alavancar de forma significativa a saúde
mental de crianças e adolescentes no Brasil. Espero que a gente possa capacitar pro-
fissionais da área de saúde mental dentro dessa visão, que possamos também divulgar
e difundir essas informaçóes, não só para o profissional da área de saúde que está em
nível secundário e terciário, mas também aquele que está lá na ponta no Posto de
Saúde de Família. Que ele possa começar a ter noçóes básicas de saúde mental dentro
do contexto da psiquiatria do desenvolvimento.

\section{Reflexōes finais}

Inicialmente, um dos objetivos do empreendimento é inserir as crianças que estão em trajetórias atípicas para trajetórias típicas. Porém, nesse caso, o normal, o típico, não foi explicado. Em nenhum momento foram explicitados os critérios de normalidade e as regras de delimitação de uma infância que a psiquiatria do desenvolvimento considere típica. No vídeo, não há uma explicação do que seria a pessoa normal para a psiquiatria do desenvolvimento. Poderíamos olhar esse ser típico, como o homem médio, aquele inserido às normas disciplinares da sociedade em que vive. Porém, as normas definem diferentes possibilidades do típico, isso depende da dimensão da norma exterior (LE BLANC, 2007, p.3950). Ainda, teria de ser levada em conta a fronteira que existe entre a pessoa típica e a pessoa ideal, que certamente não seria o mediano. Para que se pudesse compreender o que está fora da norma e da idealização de um adulto típico, seria preciso que fossem definidas as regras de educação, de disciplinarização da criança e, por fim, apresentadas justificativas para afirmar os motivos de ser essa forma de moralização a mais acertada para a manutenção da saúde mental. 
E mesmo que se defina um adulto típico, qual seria o valor de uma normalização de condutas? Essa psicologia que se pretende biológica, hoje chamada psicologia comportamental-cognitiva, é um dos instrumentos utilizados pela psiquiatria para delinear a normalização das condutas. Mesmo que os estudos, tanto genéticos como de observação comportamental, em relação ao funcionamento cerebral, localizem aspectos do comportamento, precisam ser levados em conta os muitos saberes envolvidos na compreensão do ser humano.

A situação mereceria um movimento como o que Foucault chamou de insurreição dos 'saberes sujeitados', em Em defesa da sociedade (1999), curso no Collège de France, 1975-1976. Ele entende por 'saberes sujeitados', "toda uma série de saberes que foram desqualificados como saberes não conceituais, como saberes insuficientemente elaborados: saberes ingênuos, saberes hierarquicamente inferiores, saberes abaixo do nível do conhecimento ou da cientificidade requeridos (FOUCAULT, 1999, p.12)". Para Foucault, foi por meio do reaparecimento de saberes locais, populares e do ressurgimento de saberes eruditos e novas formas de estudo da história, que esses saberes desqualificados, fizeram a crítica. Foucault fala que a ciência assujeita os saberes e define uma verdade que pode ser tão frágil em sua confirmação de verdade científica quanto os saberes não formais.

Finalizando, podemos dizer que estamos diante de um superdimensionamento do poder de solução dos fatos da vida, por uma medicina psiquiátrica e sua verdade científica, que lança mão de diagnósticos de doenças não manifestadas, baseados em comportamentos a serem contidos por psicofármacos e pela orientação de condutas. É um projeto em fase de implantação, que conta com amplo financiamento, público e privado, e credibilidade institucional para sua implantação. É uma medicalização do social e do psíquico, que serve mais à indústria da doença do que à saúde da população.

\section{Referências}

ANGELL, M. A verdade sobre os laboratórios farmacêuticos: como somos enganados e o que podemos fazer a respeito. Rio de Janeiro: Record, 2007.

CAPONI, S. et al. (Orgs.). Medicalização da vida: ética, saúde pública e indústria farmacêutica. Florianópolis: Unisul, 2010.

DSM-IV-TR. Manual Diagnóstico e Estatístico de Transtornos Mentais., 4a ed. São Paulo: Artmed, 2002. 
FOUCAULT, M. O nascimento da medicina social. In: Microfísica do poder. $5^{\mathrm{a}}$ ed. Rio de Janeiro: Graal, 1985.

. Em defesa da sociedade. Curso no Collège de France (1975-1976). São Paulo: Martins Fontes, 1999.

. Vigiar e punir, história da violência nas prisões. 36ª ed. Petrópolis: Vozes, 2007.

- Nascimento da biopolítica. São Paulo: Martins Fontes, 2008.

INSTITUTO ANTÔNIO HOUAISS. Dicionário Eletrônico Houaiss da Língua Portuguesa (Versão 1.0.10) [CD-ROM] Rio de Janeiro, Objetiva, 2006.

INSTITUTO Nacional de Ciência e Tecnologia de Psiquiatria do Desenvolvimento para a Infância e Adolescência. Nota sobre criação do INPD, 2009. Disponível em: <http://inpd. org.br/blog/2009/04/11/psiquiatria-do-desenvolvimento/>. Acesso em: 30 mar. 2011.

INSTITUTO Nacional de Ciência e Tecnologia de Psiquiatria do Desenvolvimento para a Infância e Adolescência. Vídeo institucional, s/d. Disponível em: <http://inpd.org.br/ videos/>. Acesso em: 30 mar. 2011.

LE BLANC, G. Les maladies de l'homme norma. Paris: J. Vrin, 2007.

MACHADO, R. et al. Danação da norma, medicina social e constituição da psiquiatria no Brasil. Rio de Janeiro: Graal, 1978.

MALDONATO, M. O desafio da comunicação, caminhos e perspectivas. São Paulo: Palas Athena, 2004.

MORAES, C. et al. Força-tarefa brasileira de psiquiatras da infância e adolescência. Revista Brasileira de Psiquiatria, São Paulo, v. 30, n. 3, p. 294-295, 2008.

ODA, A.M.G.R.; DALGALARRONDO, P. História das primeiras instituições para alienados no Brasil, História das Ciências da Saúde-Manguinhos, Rio de Janeiro, v. 12, n. 3, 2005.

ROHDE, L.A. et al. Quem deve tratar crianças e adolescentes? O espaço da psiquiatria da infância e da adolescência em questão. Revista Brasileira de Psiquiatria, São Paulo, v. 22, n. 1, p. 2-3, 2000.

ROSEN, G. Da polícia médica à medicina social: ensaios sobre a história da assistência médica. Rio de Janeiro: Graal, 1980.

\section{Notas}

${ }^{1}$ Força-tarefa: termo de marinha, termo militar. Grupamento de unidades de diferentes tipos, temporariamente sob comando único, para executar uma missão específica, que exige certa independência de cada unidade (HOUAISS, 2006).

${ }^{2}$ Vídeo institucional do Instituto Nacional de Ciência e Tecnologia de Psiquiatria do Desenvolvimento para a Infância e Adolescência (INPD). O vídeo completo tem a duração de 9 minutos e 7 segundos. 


\section{The task-force of developmental psychiatry}

This study analyzes the corporate video that presents the Project of the Instituto Nacional de Ciência e Tecnologia de Psiquiatria do Desenvolvimento para a Infância e Adolescência, INPD (National Institute of Science and Technology of Developmental Psychiatry for Children and Adolescents, Brazil). It aims to broaden the debate about scientific truth and methods to prevent mental illness in childhood and adolescence, through the analysis of the media material used to publicize the project. The work to prevent mental illness and early diagnosis of psychopathology has started in private schools (2010) and intends to prove its validity aiming the construction of public policies. Based on data from epidemiological research organizations, the creation of a psychiatric subspeciality is beeing articulated. We are facing a super dimension of the power of psychiatric medicine and its truth to find solutions for the facts of life, which makes use of non-expressed disease diagnosis, based on behavior, to be controlled by psychotropic drugs and conduct guidance. It is a project under implementation, with full private and public funding, and institutional credibility. Indeed, it is the medicalization of social and psychological, serving more to the disease industry than to public health.

> Key words: medicalization, psychiatry, childhood, adolescence, prevention. 